\title{
Florid Cemento-osseous Dysplasia: Report of Three Unusual Cases
}

\author{
${ }^{1}$ Fatemeh Bagheri, ${ }^{2}$ Jamileh Bigom Taheri, ${ }^{3}$ Somayyeh Azimi, ${ }^{4}$ Zahra Elmi
}

\begin{abstract}
Florid cemento-osseous dysplasia (FLCOD) is a reactive process that involves tooth-bearing areas of the jaws. Reactive or dysplastic changes in periodontal ligament have been suggested as an etiologic factor. It usually affects females and is less frequently seen in Caucasians and Asians groups. Multiple sclerotic masses involving more than one quadrant are it is radiographic appearance. Because of its benign nature periodic recall is all things to do but in symptomatic cases surgical intervention may be required.
\end{abstract}

Keywords: Florid cemento-osseous dysplasia, Radiographic image, Case report.

How to cite this article: Bagheri F, Taheri JB, Azimi S, Elmi Z. Florid Cemento-osseous Dysplasia: Report of Three Unusual Cases. Int J Experiment Dent Sci 2014;3(2):103-105.

\section{Source of support: Nil}

Conflict of interest: None

\section{INTRODUCTION}

Cemento-osseous dysplasia (COD) is an asymptomatic condition with unknown etiology and pathogenesis; which is characterized by disorder in bone production and cementum like tissue. World Health Organization has been categorized COD into three subgroups depending on their location and radiographic features: periapical, focal and florid COD. ${ }^{1,2}$ Middle-aged black women are most commonly affected, although Caucasians and Asians may be involved too. ${ }^{3}$ Florid cemento-osseous dysplasia (FLCOD) is a very rare lesion and may be familial in some cases. ${ }^{4,5}$ Radiographically this condition presents as multiple sclerotic masses which a radiolucent rim may surround them and the lesion occur within the alveolar bone of two or more quadrants. ${ }^{3,6}$ In asymptomatic patients follow-up and periodic radiographic examination with prophylaxis and good oral hygiene are the best considerations. ${ }^{7}$ If the sclerotic masses expose in the oral

\footnotetext{
${ }^{1,4}$ Resident, ${ }^{2}$ Professor, ${ }^{3}$ Assistant Professor

${ }^{1-4}$ Department of Oral Medicine, Dental School, Shahid Beheshti University of Medical Sciences, Tehran, Iran
}

Corresponding Author: Somayyeh Azimi, Assistant Professor, Department of Oral Medicine, Dental School, Shahid Beheshti University of Medical Sciences, Tehran, Iran, Phone: 00982122403010, e-mail: somayyeh_azimi@sbmu.ac.ir cavity, the patient will complaint of dull pain or drainage 5 in such cases surgical intervention be advised. ${ }^{6}$ This paper describes three cases of FLCOD two asymptomatic and one symptomatic that patient presented with pain and pus drainage of the mandibular ridge because of exposure in the oral cavity.

\section{CASE REPORTS}

\section{Case 1}

A 61-year-old female was referred to our department with the diagnosis of bilateral bony lesions of the mandible. Her chief complaint was ill-fitting mandibular denture, pain and pus drainage since 1 year ago. She stated that her dentures were fabricated 3 years ago and after 2 years she was not able to use them more. Her dentist had visited her 1 year ago because of pain in right side of the mandible and panoramic view was requested. In habitual history she mentioned the history of tobacco use for some years. She had high blood pressure and diabetes mellitus and was receiving captopril, amlodipine and glibenclamide as well. Intraoral examination revealed a normal and intact mucosa in the left side and a sinus with pus drainage in the right side of the mandible. In panoramic view multiple radiopaque lesions in different size without radiolucent rim above the mandibular canal were seen in the left side and a radiopaque mass in the right side that was exposed to the oral cavity due to alveolar bon resorption (Fig. 1). A diagnosis of FLCOD was established considering radiographic image and the patient referred to the surgeon for further management. Partial osteotomy and curettage was done and patient advised to periodic recalls.

\section{Case 2}

A 34-year-old female patient was referred to our department with chief complaint of bony lesions in her jaw about 4 months before referral. For the first time, her dentist requested panoramic view due to dental evaluation and then bilateral mixed lesions was detected incidentally. Bony lesions were diagnosed and the patient was referred to dental college for further assessment. She was a healthy female with no medical complication. Intraoral examination revealed multiple extracted teeth due to poor oral hygiene (f. panoramic view showed a large mixed lesion with opacity of varying density extending from tooth number 


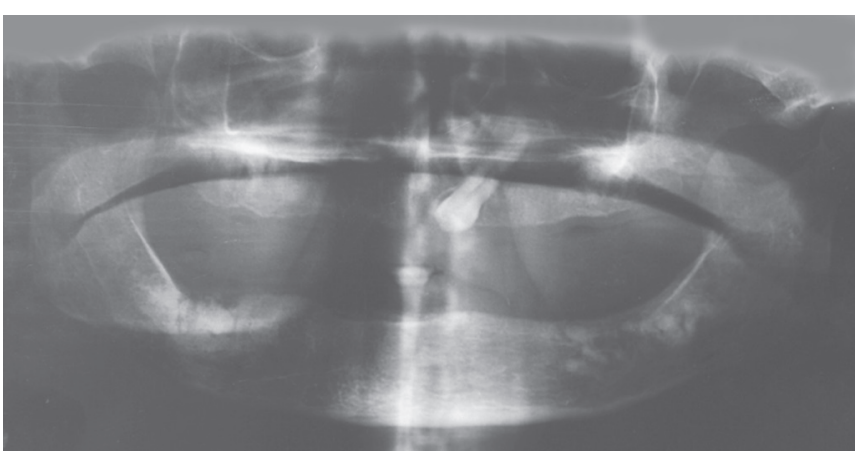

Fig. 1: Panoramic view (Case 1)

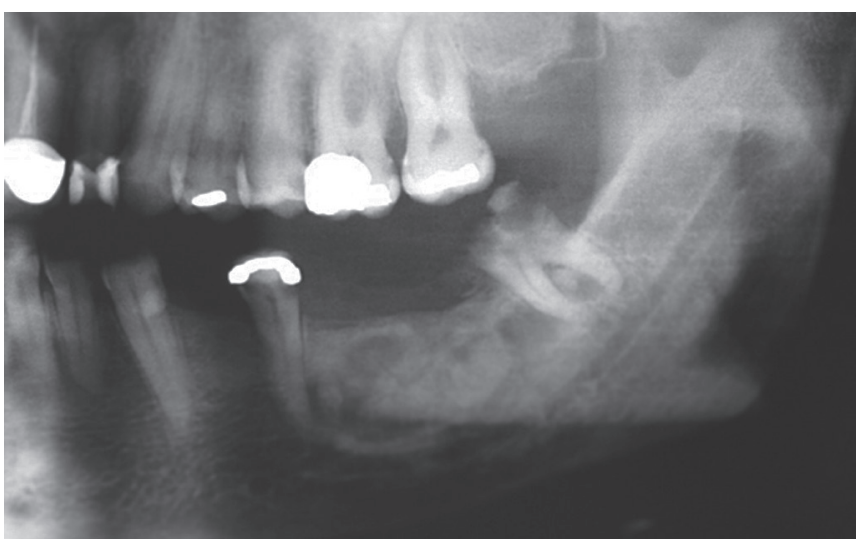

Fig. 3: Panoramic follow-up view (Case 2)

44 to tooth number 48 in the right side of the mandible. Another radiolucent lesion extending from tooth no. 35 to tooth no. 38 was detected in the left side of mandible. Both lesions were above the mandibular canal (Fig. 2). According to radiographic feature the diagnosis of FLCOD was established. Tooth number 37 was hopeless because of mobility and furcation involvement so it was extracted and antibiotic and analgesic was used. Patient reported severe pain after extraction and the surgeon was requested a panoramic. The lesion was more mature and there was nothing wrong with the extraction site (Fig. 3).

\section{Case 3}

A 36-year-old female came to our department for routine dental examination. Medical and habitual history was not remarkable. Intraoral examination revealed multiple missing teeth in all four quadrants that were extracted because of poor prognosis and financial problems and she wanted to replace them with partial denture. A panoramic view was requested for further evaluation. In panoramic view there were an opaque mass with well-defined border in the posterior segment of the right side of the mandible and a mixed lesion in the left side mesial to the second molar that was very near to the alveolar ridge (Fig. 4). Both of them were above the mandibular canal. Finally the diagnosis of FLCOD was established and the patient was advised to periodic recall.

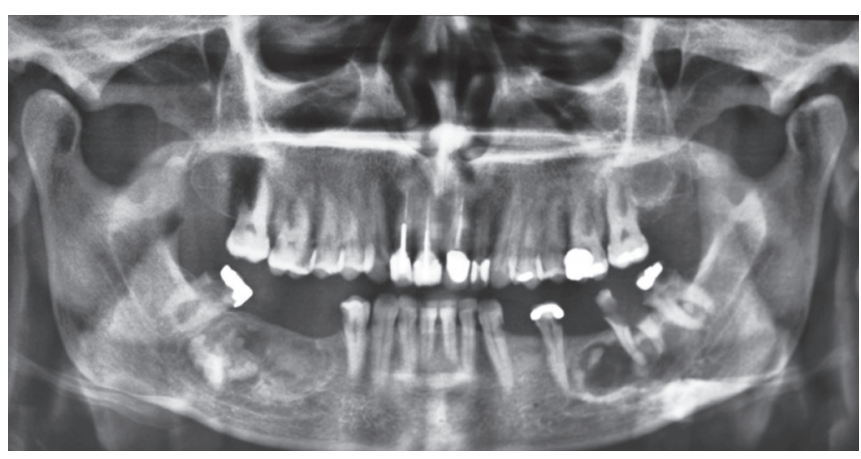

Fig. 2: Panoramic view (Case 2)

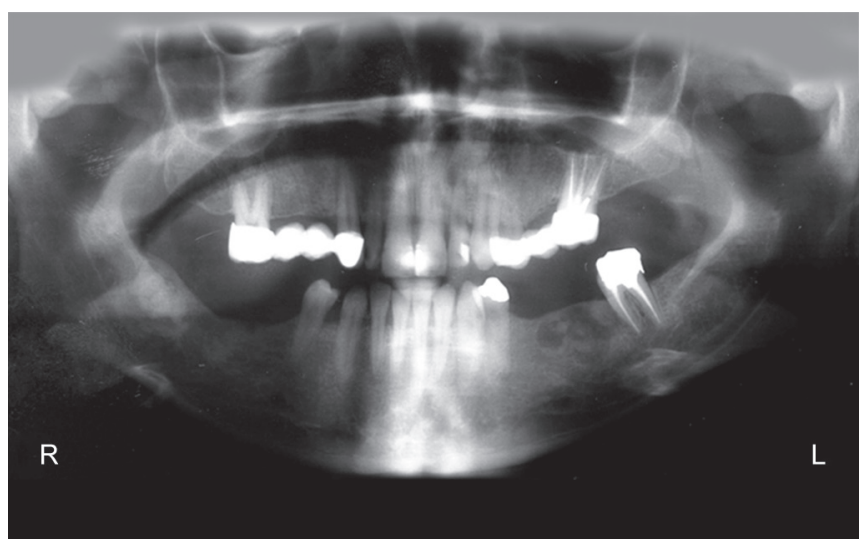

Fig. 4: Panoramic view (Case 3)

\section{DISCUSSION}

We report 3 cases of FLCOD in different ages in females. Melrose et al first introduced FLCOD in $1976 .{ }^{2}$ Florid cemento-osseous dysplasia is a reactive process with involvement of tooth bearing areas of the jaws. ${ }^{6}$ The word florid explains extensive manifestation. ${ }^{2}$ The etiology is not clearly understood, but reactive or dys- plastic changes in periodontal ligament have been suggested as the reason. ${ }^{4}$ Depending on the degree of maturation it has three radiographic features: lucent, mixed and opaque lesion, but the characteristic appearance is diffuse radiopaque masses. Most cases occur bilaterally in the mandible and usually with no cortical expansion or pain. ${ }^{2,5}$ Histopathology changes consist of replacement of bone by connective tissue matrix with varying degree of mineralization. ${ }^{8}$

In a systematic review MacDonald and Jankowski reported a mean age of 47 to 49 years old and a predilection for female gender (97\%) in FLCOD patients. ${ }^{9}$ In this study, our first case was a woman in her seventh decade of life that is higher than normal range that have been mentioned for FLCOD and two other cases were younger than normal range. Although these lesions often discover accidentally and are asymptomatic, ${ }^{4}$ our first case complained from pain and pus drainage since 1 year ago.

Diffuse masses with varying degree of maturation are the radiographic appearance of FLCOD. ${ }^{4}$ Our cases were compatible with these criteria and had bilateral mandibular 
opaque lesions. Since FLCOD lesions are prone to infection because of insufficient blood circulation in the area and subsequent osteomyelitis, biopsy and surgical intervention is not recommended ${ }^{2}$ unless secondary infection superimpose, as in our case that the lesion exposed in the oral cavity and infected due to alveolar ridge resorption. For asymptomatic lesions regular follow-up is indicated. ${ }^{2,7}$

Florid cemento-osseous dysplasia should not be misdiagnosed as Paget's disease, chronic diffuse osteomyelitis and Gardner syndrome. Because of no other skeletal involvement, tooth anomaly and pain or swelling, FLCOD can be differentiated from these conditions. ${ }^{3}$

\section{CONCLUSION}

Although, FLCOD usually presents as an asymptomatic lesion, In case of infection and inflammation, it is a complicated condition to cure because of increased bone density and decreased vascularity, so its diagnosis is valuable to avoid these complications. Dentistry is varying with induction of modern science to practice dentistry. ${ }^{10}$

\section{ACKNOWLEDGMENT}

Authors would like to thank the Oral Medicine Department of Shahid Beheshti University of Medical Sciences, Tehran, Iran, for its immense support to refer cases in completing this study.

\section{REFERENCES}

1. Komabayashi T, Zhu Q. Cemento-osseous dysplasia in an elderly Asian male: a case report. J Oral Sciences 2011;53: 117-120.

2. Komali G, Vinitha KB, Rao M. Cemento-osseous dysplasia: a case report. Int J Dent Case Reports 2012;2(5):42-46.

3. Sentürk MF, Kestane R, Yakar EN, Keskin A. Florid cementoosseous dysplasia: a rare case report. Case Reports in Dentistry Volume 2013, Article ID 946583, 4 pages.

4. Imanimoghaddam M, Tafakhori Z. Florid cemento-osseous dysplasia: report of three symptomatic cases. Iran J Radiol 2010; 7(3):189-192.

5. Gunduz K, Avsever H, Karacayli Ü, Şenel B, Piskin B. Florid cemento-osseous dysplasia: a case report. Braz Dent J 2009;20(4): 347-350.

6. Pereira T, Tamgadge AP, Chande MS, Bhalerao S, Tamgadge S. Florid cemento-osseous dysplasia: case report of a rare entity. Int J Contemp Dentist 2011;2:38-40.

7. Babaria U, Patel H, Pandya H, Dewan H, Bhavasar B, Thakkar D, et al. florid osseous dysplasia: a case report. J Dent Sci 2011; 2(2):10-11.

8. Cyntia Helena Pereira de Carvalho, de Araújo Lima, Joabe dos Santos Pereira, Ana Miryam Costa de Medeiros, Éricka Janine Dantas da Silveira. Florid cemento-osseous dysplasia and osteomyelitis: a case report of a simultaneous presentation. Rev Odonto Cienc 2012;27(2):166-169.

9. MacDonald-Jankowski DS. Florid cemento-osseous dysplasia: a systematic review. Dentomaxillofac Radiol 2003;32(3): 141-149.

10. Saini R. Ozone therapy in dentistry: A strategic review. J Nat Sc Biol Med 2011;2:151-153. 